\title{
La siglaison en langue médicale et problèmes de traduction
}

\section{Sylvie Monin}

\section{(2) OpenEdition}

\section{Journals}

Édition électronique

URL : http://journals.openedition.org/asp/4264

DOI : 10.4000/asp.4264

ISSN : 2108-6354

\section{Éditeur}

Groupe d'étude et de recherche en anglais de spécialité

\section{Édition imprimée}

Date de publication : 1 octobre 1993

Pagination : 29-53

ISSN : 1246-8185

\section{Référence électronique}

Sylvie Monin, «La siglaison en langue médicale et problèmes de traduction », ASp [En ligne], 2 | 1993,

mis en ligne le 26 février 2014, consulté le 01 mai 2019. URL : http://journals.openedition.org/ asp/4264 ; DOl : 10.4000/asp.4264

Ce document a été généré automatiquement le 1 mai 2019.

Tous droits réservés 


\title{
La siglaison en langue médicale et problèmes de traduction
}

\author{
Sylvie Monin
}

\section{Introduction}

1 Avant de spécifier les objectifs de cette communication, je définis les termes employés.

\section{Le sigle spécialisé}

2 Au sens strict, c'est l'initiale en majuscule d'un seul mot ou un ensemble d'initiales majuscules de plusieurs mots, fréquemment utilisés en langue spécialisée. Par exemple :

CT : Capacité Totale

A : Artery

3 Au sens large, cela peut être la juxtaposition de consonnes composant en partie un mot.

Par exemple :

svt : suivant

EDTA : edetate (éthylène-diamine-tétraacétique)

\section{La siglaison}

4 C'est le processus de formation d'un sigle, c'est-à-dire la réduction d'un mot ou d'un groupe de mots à des initiales. Par exemple :

GH : Growth Hormone (hormone de croissance)

\section{L'abréviation}

5 C'est le retranchement de plusieurs lettres dans un mot. Sa présentation est parfois caractérisée par des points placés entre les lettres et par l'emploi très fréquent de lettres minuscules. La (les) lettre(s) peut (peuvent) être associée(s) à un ou plusieurs chiffres. Par exemple :

A2 : Aortic second sound (second bruit aortique)'

Q CO2.: Quantité de gaz carbonique (Carbon Dioxide - CO2.) éliminée par les tissus

et par heure.

Elle peut être variable. Par exemple : 
acdts : (en français) accidents

\section{Le symbole chimique}

6 C'est la lettre (ou le groupe de lettres) utilisée(s) pour désigner la masse atomique d'un élément. Par exemple :

A1 : Aluminium (seule la première lettre est une majuscule)

\section{La troncation}

7 C'est le processus de suppression d'une syllabe ou d'une lettre ou de plusieurs lettres d'un mot. La troncation peut être postérieure (apocope), antérieure (aphérèse) et même double dans cet exemple :

Flu : Influenza

\section{Les éponymes}

Ce sont des noms propres utilisés pour désigner des syndromes ou des maladies. Par exemple :

GTS : Gilles de la Tourette's Syndrome

\section{L'acronyme}

9 C'est un mot formé en combinant la première lettre ou des lettres d'un mot ou d'une phrase. Par exemple :

ELISA : Enzyme-Linked-Immunoabsorbent Assay

10 Les lettres majuscules de l'acronyme peuvent devenir minuscules quand l'acronyme est reconnu et accepté par la communauté scientifique internationale. Il est donc considéré comme un nom commun dans ce cas.

11 Dans cet article, j'ai l'intention d'examiner si l'abondance des abréviations anglaises provient soit de l'emprunt du sigle anglais qui prend le pas sur la création d'un sigle en français, soit du caractère fort explicite du sigle anglais. Dans ce cas, il n'est pas traduit en français, car la communauté scientifique le connaît déjà et l'a assimilé dans sa terminologie médicale. De plus, seront évoqués au cours de cette étude, des problèmes lexicaux liés à la traduction des sigles en français et en anglais de la médecine.

\section{Méthode}

12 Cette étude contrastive de la siglaison en langue médicale a été réalisée à partir de « condensés d'articles » (abstracts) figurant dans les quarante-cinq exemplaires de revues médicales publiées entre 1989 et 1992 et destinées aux professionnels. Aucun critère n'a guidé le choix des revues traitant de chirurgie, psychiatrie, cardiologie, génétique, urologie, radiologie, biologie clinique, pédiatrie, rhumatisme, gynécologie, etc. Un haut niveau de spécificité scientifique a seulement été retenu.

Les textes initiaux des abstracts ont été considérés de façon comparée en les mettant en regard avec le texte traduit soit en français soit en anglais, et pour cinq cas en espagnol. Seuls les abstracts ont été étudiés car ils comportent des sigles définis pour la première fois par le scientifique-rédacteur de l'article.

14 Suivent quelques extraits des règles générales de rédaction concernant l'abstract et les formes abrégées :

Standard abbreviations may be used without explanation. All other abbreviations must be inserted in parentheses following the first use of the term. Abbreviations defined in the abstract should not again be defined in the body of the text. (Council 
of Biology Editors Style Manual, chapter 12 Instructions to Authors for Current Microbiology 23/2, August 1991)

Terms appearing frequently within a paper may be abbreviated but should be spelled out in full at the first citation with the abbreviation following in parentheses. (Annals of Hand and Upper Limb Surgery 10/6 1991. Instructions for authors, 498)

Les abréviations en trop grand nombre sont à éviter. Celles qui n'entreraient pas dans les usages internationaux doivent être définies par une note en bas de page dès la première apparition de l'une d'entre elles. (Annales de Biologie Clinique 1991, 49/7)

Les abréviations citées doivent être conformes à celles de l'Index Medicus. Les abréviations d'unités de mesure et de symboles chimiques doivent être conformes aux normes internationales. Le manuscrit doit comporter le minimum d'abréviations: celles-ci doivent être indiquées à leur premier emploi, après l'expression ou le mot qu'elles abrègent, en lettres uniquement. Ex : Rheu Fev pour Rheumatic Fever. (La Revue du rhumatisme et des maladies ostéo-articulaires 1992, 6)

Cependant, il faut signaler la limite méthodologique suivante: les professionnelsrédacteurs ne respectent pas toujours les pratiques courantes mentionnées ci-dessus ou ne les adoptent pas. Pour illustrer cette limite, voici ces directives extraites de la Revue française de gynécologie et d'obstétrique (1992, 87/1):

Les abréviations dans le texte sont interdites, sauf celles d'usage international. Résumé de 100 à 250 mots, sans abréviation ni référence, précisant les objectifs, la méthodologie, les principaux résultats et les conclusions. Le contenu du résumé ne doit s'écarter en rien du contenu de l'article.

Par conséquent, le lecteur, désireux de comprendre le texte du condensé de l'article ainsi que le texte de l'article doit surtout maîtriser sa connaissance des sigles internationaux nouveaux et des sigles empruntés à d'autres disciplines. Voici un exemple pour illustrer ce propos :

L'existence d'un système «HIS » est un préalable pour le bon fonctionnement d'un système « PACS ».

Dans cet exemple, l'auteur de la phrase n'a pas expliqué le sens des sigles utilisés. Cela pose un problème de compréhension au lecteur peu familier des sigles internationaux relatifs à l'imagerie médicale ou empruntés à une autre discipline.

Le sigle « HIS » signifie « Hospital Information Systems » et désigne un système concernant la saisie et l'archivage de données médicales, soit écrites, soit chiffrées.

Le sigle «PACS » correspond à l'expression "Picture Archiving and Communication Systems » et désigne un système de câblage en réseau de communication et de transport d'images : c'est-à-dire, que ces réseaux facilitent la communication des différentes sources d'images médicales avec les différents sites d'utilisation (hôpitaux, laboratoires), et assurent l'archivage à court, moyen et long terme de ces données.

\section{Résultats et présentation de problèmes de traduction liés a l'utilisation de la siglaison en langue médicale}

20 À partir du corpus d'abstracts médicaux mentionnés auparavant, 204 sigles anglais contre 158 sigles français ont été relevés. Comment peut-on expliquer cet écart de 46 sigles ? La communauté scientifique médicale anglaise crée-t-elle réellement plus de sigles que celle 
française? La communauté scientifique médicale française emprunte-t-elle les sigles anglais sans même les traduire? La nature même de la langue française ne facilite-t-elle peu la création de sigles? Les sigles anglais ont-ils un caractère assez explicite qui les rend empruntables sans les traduire par la communauté des scientifiques français?

Tableau 1. Répartition des sigles français et anglais

\begin{tabular}{|l|l|l|}
\hline & corpus GB - F & F - GB \\
\hline Sigles n'existant qu'en français & 1 cas $(0,5 \%)$ & 67 cas $(42,40 \%)$ \\
\hline Sigles existant à la fois en anglais et en français & 78 cas $(38,3 \%)$ & 78 cas $(49,3 \%)$ \\
\hline Sigles n'existant qu'en anglais & 90 cas $(44,2 \%)$ & 2 cas $(1,3 \%)$ \\
\hline Sigles empruntés à l'anglais par le français & 1 cas $(0,5 \%)$ & 67 cas $(42,4 \%)$ \\
\hline Sigles empruntés au français par l'anglais & 34 cas $(16,5 \%)$ & 7 cas $(4,5 \%)$ \\
\hline TOTAL & 204 cas & 158 cas \\
\hline
\end{tabular}

21 À partir du tableau 1, on remarque surtout l'abondance des sigles n'existant qu'en anglais ( $44,2 \%$ des cas), et la relative importance des sigles anglais empruntés en français (16,5\% des cas), en allant de l'anglais vers le français. Dans le sens français-anglais, on peut mentionner que le nombre de sigles n'existant qu'en français ( $42,4 \%$ des cas) est inférieur à celui prédominant des sigles existant à la fois en français et en anglais (49,3\% des cas).

À l'inverse et dans le sens anglais-français, le nombre de sigles n'existant qu'en anglais ( $44,2 \%$ des cas) dépasse celui des sigles existant à la fois en français et en anglais ( $38,3 \%$ des cas).

En outre, il serait intéressant d'examiner pourquoi

- autant de sigles n'existent qu'en anglais ( $44,2 \%$ des cas)

- moins de sigles n'existent qu'en français (42,4\% des cas)

- note-t-on si peu d'emprunts de sigles français en anglais (0,5 \% des cas) et

- pourquoi compte-t-on un pourcentage plutôt faible d'emprunts de sigles anglais en français (de $4,5 \%$ à $16,5 \%$ des cas)?

\subsection{L'abondance des sigles anglais}

Cette abondance semble due à la facilité qu'ont les scientifiques anglais de former de longues compositions de mots ou "supercomposés» afin de transmettre le plus succinctement possible l'information scientifique. À l'aide des exemples suivants, on peut souligner que le français, à défaut de ne pouvoir concentrer les données médicales, est obligé de les expliciter pour préserver la communication :

LRTI : Lower Respiratory Tract Infection (Infection de l'Arbre Respiratoire Inférieur)

HDL : High Density Lipoprotein (Lipoprotéine de Haute Densité)

Face au problème de la complexité des compositions de mots en anglais, le traducteur emprunte le sigle anglais soit en le traduisant à l'aide de prépositions et d'articles 
supplémentaires comme dans les exemples précédents, soit en empruntant la totalité de l'expression anglaise et le sigle anglais comme le prouvent les exemples suivants :

TSH : Thyroid-Stimulating Hormone

fratrie) suivants : suivants :

Le tiret marque une relation établie entre "thyroid» et "stimulating». La forme -ing rappelle une structure plus profonde - a hormone that stimulates the thyroid gland.

ELISA : Enzyme-linked immunoadsorbent assay

AFTA : Autonomously-Functioning Thyroid Adenomas

PAF : Platelet Aggregation Factor

TBG : Thyroxine-Binding Globulin

Même dans les cas de troncation rencontrés en anglais, on note que le français développe l'information médicale. Ce sont les cas de l'apocope en anglais comme le montrent les exemples suivants :

SIBs : Siblings (apocope avec marque du pluriel maintenue) frères et sœurs (ou

Sep : separated (séparé)

Nyst : Nystagmus en français

Dans les exemples suivants, le français a développé les expressions en rajoutant des mots :

$\mathbf{H}$ and $\mathbf{P}:$ History and Physical (examination)

SF et SP : Signes Fonctionnels et Signes Physiques (interrogatoire et examen)

SBP and DBP : Systolic and Diastolic Blood Pressures

On peut traduire cette concentration de l'information en anglais à l'aide du mot Pressures de la manière suivante : pression artérielle systolique et tension diastolique.

FDH : Familial Dysalbuminemic Hyperthyroxinemia

En français, on emprunte le sigle FDH et l'on traduit cette expression par " l'H yperthyroxinémie Familiale » avec « Dysalbuminémie ». À une exploitation « facile » de la voyelle de liaison « 0 » et du trait d'union en anglais, peuvent correspondre un emprunt, un calque, un développement de l'expression en français comme le prouvent les exemples

IFMA : ImmunoFluorometric Assay (emprunt en français)

EID : ElectroImmunoDiffusion (emprunt en français)

RAST : Radio-AllergoSorbent Test (emprunt en français sous la forme suivante:

Radio Allergo Sorbent

RIA : RadioImmunoAssay; Radio-Immuo-Essay (calque); RadioImmunoDosage ;

Méthode Radio-Immunologique (développement)

\subsection{Les sigles en français moins nombreux}

Les sigles semblent moins nombreux en français qu'en anglais pour lever toute ambiguïté lexicale ou la création de faux-amis.

Par exemple, en anglais :

OS : Opening Snap (claquement d'ouverture de la mitrale)

o. S., o. s. : Oculus Sinister ; left eye ; ('œil gauche)

Il ne faut pas confondre ces abréviations avec les suivantes en français :

OS : bouche, ouverture (mouth, opening - pas d'abréviation en anglais)

OS : Occipito-sacrée (occipito-posterior position) (position)

En français, on note une certaine aversion pour les surcomposés, à l'aide des exemples 
DSA : Digital Substraction Angiography (angiographie numérisée, pas d'abréviation en français) (emprunt du sigle anglais)

EGF : Epidermal Growth Factor (facteur de croissance épidermique, emprunt du sigle anglais)

B1 cult : Blood Culture

En français, on évite l'apocope et la juxtaposition de mots en utilisant le préfixe hémo- : hémoculture, d'où l'absence d'abréviations.

GB-ca: GallBladder Carcinoma (cancer de la vésicule biliaire [développement et aucune abréviation en français]

IGF-BP : Insulin-like growth factor-binding protein (emprunt en français $d u$ surcomposé savant avec like et binding)

En français médical, on emprunte des sigles anglais reconnus par la communauté scientifique internationale. Par exemple,

SPECT : Simple Photon Emission Completed Tomography [tomographie numérisée à émetteur gamma]

UNICEF : United Nations Children's Emergency Fund [Fonds des Nations Unies pour l'Enfance]

Produits par les lymphocytes, le Granulocyte-Monocyte Colony Stimulating

Factor [GM-CSF] et l'interleukine 3 agissent sur plusieurs catégories de cellules.

Selon la technique BSA [Biotine-Streptavidine avec marquage à la peroxydase]

According to the BSA Technique (Biotine-Streptavidine with peroxidase labelling

Le français n'utilise pas toujours un acronyme correspondant à une expression existante en anglais, et vice-versa. Par exemple,

ANOVA : Analysis of Variance (les premières lettres sont imbriquées - emprunt en français)

TRIAC : Triiodothyroacetic acid

DRILA : Douleur Réveillée à l'injection Légèrement Appuyée.

ANDEM : Agence nationale pour le développement de l'évaluation médicale

Les sigles d'organisations nationales ne sont pas seulement empruntés dans la langue d'arrivée mais aussi ceux d'organisations internationales. Par exemple, un équivalent est donné

IPRS : Conféderation Internationale de Chirurgie Plastique et Reconstructive

CHG : Centres Hospitaliers Généraux (non-teaching hospitals)

CHU : Centre Hospitalo-Universitaire; Centre Hospitalier Universitaire (teaching

hospitals ; teaching hospital group, university hospital)

CNRS: Centre national de la recherche scientifique (French National Science Council)

Cependant, il faut se méfier de la polysémie des acronymes. Seul le contexte peut aider à lever les ambiguïtés. Par exemple :

SIDA : Sacro-illiaque Droite Antérieure (RSA : Right SacroAnterior

SIDA : Syndrome Immuno Déficitaire Acquis (Gay Syndrome)

AIDS : Acquired ImmunoDefficiency Syndrome

AID : Artificial Insemination with the Donor's semen (IAD : Insémination Artificielle avec sperme du Donneur ou sperme congelé de Donneurs).

Le scientifique français n'a pas les mêmes habitudes que son collègue anglophone dans la manière d'abréger des mots ou des expressions. Par exemple :

DA : Diagnostic Accuracy (le précision diagnostique - pas d'abréviation en français).

DEL (ou del) : Deletion (délétion - pas d'abréviation en français)

NC : No change (échec [dans une réponse] - pas d'abréviation en français) 
De plus, les scientifiques anglophones emploient de manière habituelle des éponymes. Les noms des savants sont abrégés. Par exemple :

J S : JOUBERT'S Syndrome (le syndrome de JOUBERT)

Pap Smear: PAPANICOLAOU test (frottis cervical ou vaginal)

US : USHER'S Syndrome (le syndrome de USHER).

Cependant, il faut signaler que l'emprunt d'un sigle anglais peut entraîner un problème d'interprétation ou un problème d'identification sémantique. Dans l'exemple suivant, le traducteur a préféré donner un équivalent plutôt que d'emprunter les sigles :

- en allant de l'anglais

A full ROM of the PIPJ was regained in 10 cases.

PIPTJ : Proximal Interphalangeal Joint (articulation proximale interphalangienne)

ROM : existence de plusieurs sens : Read Only Memory (mémoire morte, mémoire

fixe) ; rupture of membrane ; range of motion

- vers le français :

La récupération de l'amplitude a été complète dans 10 cas

- vers l'espagnol :

La récupéracion de la amplitude fue completa en 10 casos.

La traduction des sigles aurait posé un problème de compréhension au lecteur.

\subsection{Juxtaposition de mots savants}

La faible importance des emprunts des sigles français en anglais semble due à la rareté de longues juxtapositions de mots savants en français par souci de concision et à des raisons sémantiques, par exemple, en allant du français vers l'anglais :

Les auteurs rapportent leur expérience de l'utilisation du système de gestion de bases de données médicales (LIED), support des dossiers médicaux informatisés THESEE et ARTEMIS.

LIED : Langage Interactif pour l'Exploitation des Données

Il s'agit d'un logiciel destiné à la gestion de bases de données évolutives.

The authors report their experience in using the LIED - medical data processing system - which forms the basis for the computerized THESEE and ARTEMIS programs.

Les éponymes sont empruntés en anglais. Par exemple,

BCG : Bacille CALMETTE-GUERIN

Les scientifiques anglais et français n'ont pas les mêmes habitudes d'abréger les mots ou les expressions :

cc : Corps Calleux (the corpus callosum - pas d'abréviation en anglais)

tr : troubles (disorders, - pas d'abréviation en anglais)

$\mathbf{v}$ :ventriculaire (ventricular - pas d'abréviation en anglais)

SEA : Syndrome d'Enthésiopathie et Arthrite séronégative (seronegative enthesopathy and arthropathy syndrome - pas d'abréviation en anglais).

\subsection{L'importance plutôt faible des emprunts des sigles anglais en français}

Pour des raisons de lisibilité du texte, le scientifique français évite d'emprunter des sigles anglais. Il leur préfère des sigles équivalents. Par exemple :

ALAT : Alanine-Amino-Transférase est l'équivalent du sigle anglais 
SGPT : Transaminase Glutamino-Pyruvique sérique Serum Glutamic Pyruvic T ransaminase

AMM : Autorisation de Mise sur le Marché

PL : Product Licence [in US)

ASAT : Aspartate-Amino Transférase

SGOT: Serum Glutamic Oxaloacetic Transaminase (transaminase glutamique oxalacétique du sérum)

BK : Bacille de $\mathrm{KOCH}$

TB : Tubercle Bacillus

TSH : Thyroid-Stimulating Hormone

TSH : La thyréostimuline

Les sigles anglais empruntés sont explicités en français :

FTA test: Fluorescent Treponeme Antibody (test d'immunofluorescence pour le diagnostic de la syphilis)

IMA : Internal Mammary Artery (graft) pontage coronarien avec greffon mammaire HMG : Human Menopause gonadotropin Menopausal (Hormone gonadotrope humaine de la ménopause [emprunt du sigle en français])

STH ou GH: Somathormone, growth hormone, hormone hypothysaire de croissance (dosée dans le sang)

\section{Conclusion}

En langue médicale, l'emprunt du sigle anglais ne semble pas prendre le pas sur la création d'un sigle en français pour des raisons de compréhension et de communication de l'information scientifique.

L'abondance des abréviations anglaises n'est pas seulement favorisée par leur caractère explicite (les rendant ainsi empruntables et assimilables dans la langue d'accueil), mais aussi par la facilité de créer des surcomposés en anglais, et par l'exploitation de l'apocope, du trait d'union et de la voyelle de liaison « 0 ». Ceci facilite donc le processus de concentration de l'information médicale en anglais.

51 En français, on semble plus limiter l'emploi des sigles pour lever toute ambiguïté lexicale et pour éviter la création de faux-amis parmi les sigles, malgré le caractère explicite du contexte. On évite la juxtaposition longue des mots en trouvant soit un équivalent au sigle anglais emprunté (c'est le développement en français), soit en empruntant le sigle et son explication en anglais. En outre, le français utilise plus timidement l'acronyme pour communiquer plus rapidement l'information. On a pu noter que les traducteurs préfèrent donner un développement en traduisant en français le sigle anglais pour limiter les problèmes de compréhension liés à l'emprunt du sigle anglais et même trouver un équivalent au sigle concerné.

52 Enfin, il faut signaler que les communautés scientifiques, anglophone et francophone, n'ont pas les mêmes habitudes dans la manière d'abréger des mots ou des expressions. Seuls les sigles anglais, de nature internationale, sont plus facilement empruntés et assimilés en français. 


\section{ANNEXES}

\section{Annexe 1. Compte rendu des discussions}

À la suite de la communication, le débat a permis de soulever le problème du « métissage culturel » fort regrettable pour la communauté scientifique française. Cependant, il a été noté que l'importance de ce métissage peut varier selon les disciplines (par exemple : les informaticiens sont plus influencés par les « conventions anglo-saxonnes » que les médecins ne le sont en raison d'une « forte tradition française dans leur discipline ») et la langue (le français médical semble plus résister à ce type d'influence que l'espagnol).

Il a été remarqué que plus l'influence de la communauté scientifique anglophone est forte, plus le traducteur ne cherche pas à traduire le sigle, surtout en informatique et en électronique. À l'oral, les scientifiques ont besoin de donner à la fois le sigle et sa signification alors qu'à l'écrit, les rédacteurs spécialistes n'expliquent pas toujours le sigle utilisé. En l'absence de «fléchage situationnel » ou d'« étoffement », se pose un problème de compréhension, notamment pour les sigles internationaux récents et selon les disciplines. À ce problème, s'ajoute celui du temps de repérage de ces sigles nouveaux par la communauté de spécialistes en fonction de l'accélération des progrès techniques (par exemple, l'apport de l'électronique a « révolutionné » l'imagerie médicale). Enfin, au piège sémantique, l'importance d'une bonne coopération linguiste (ou traducteur)/ spécialiste a été mise en avant afin de mieux confronter toutes les interprétations possibles des sigles, notamment empruntés à d'autres disciplines.

\section{Annexe 2. Sources (fichier)}

Annexe 3. Liste des acronymes, des abréviations et des symboles chimiques rencontrés en majeure partie dans les résumés médicaux (fichier)

\section{RÉSUMÉS}

Cette communication pose principalement les deux questions suivantes : peut-on expliquer le nombre élevé d'acronymes et d'abréviations en anglais médical par une création lexicale active ou empruntons-nous ces formes abrégées au lieu de leur donner des équivalents en français médical ? Est-ce que ce phénomène est causé par leur degré élevé d'explicitation entraînant leur intégration rapide (sans traduction) en français médical?

This article poses the two following questions: Can the high number of English acronyms and abbreviations be explained by an active lexical creation in medical English or by their borrowing instead of creating the corresponding ones in medical French? Is this phenomenon caused by their high degree of clarifying involving their quick integration without being translated into medical French? 
INDEX

Mots-clés : abréviation, apocope, éponyme, résumé, sigle

Keywords : abbreviation, acronym, abstract, eponym

\section{AUTEUR}

\section{SYLVIE MONIN}

Université Lyon 1. monin-badey@wanadoo.fr 